\title{
High Repetition, TEMoo mode, Compact Sub-Nanosecond 532nm Laser
}

\author{
Dong-Dong Meng ${ }^{13,4,5}$, Tian-Qi Wang ${ }^{1}$, Mi Zhou ${ }^{2}$, Zhan-Duo Qiao', Xiao-Long Liu' ${ }^{1}$, Zhong-Wei Fan ${ }^{1,3 *}$
}

1 Aerospace Information Research Institute, Chinese Academy of Sciences, Beijing 100094, China; mjf09@163.com (D.-D.M.); wangtq@aircas.ac.cn (T.-Q.W.); qiaozd@aircas.ac.cn (Z.-D.Q.); liuxiaolong@aoe.ac.cn (X.-L.L.)

2 Institute of Microelectronics of the Chinese Academy of Sciences, Beijing 100029, China; milyzhou@126.com (M.Z.)

3 School of Optoelectronic, University of Chinese Academy of Sciences, Beijing 100049, China

4 University of Chinese Academy of Sciences, Beijing 100049, China

5 HARGLO Applied Laser Technology Institute Co., Ltd, Tianjin 300000, China

* Correspondence: fanzhongwei@aoe.ac.cn (Z.-W.F.)

\begin{abstract}
As a critical transmitter, the compact $532 \mathrm{~nm}$ lasers operating on high repetition and narrow pulse widths have been used widely for airborne or space-borne laser active remote sensing. We developed a free space pumped TEMoo mode sub-nanosecond $532 \mathrm{~nm}$ laser that occupied a volume of less than $125 \mathrm{~mm} \times 50 \mathrm{~mm} \times 40 \mathrm{~mm}$ (0.25 liters). The fundamental $1064 \mathrm{~nm}$ laser consists of a passively Q-switched composite crystal microchip laser and an off-axis, two-pass power amplifier. The pump sources were two single-emitter semiconductor laser diodes (LD) of $808 \mathrm{~nm}$ with a maximum continuous wave $(\mathrm{CW})$ power of $10 \mathrm{~W}$ each. The average power of fundamental $1064 \mathrm{~nm}$ laser was $1.26 \mathrm{~W}$ with the laser operating at $16 \mathrm{kHz}$ repetition rates, and $857 \mathrm{ps}$ pulse widths. Since the beam distortion would be severe in microchip lasers in terms of the increase in heat load, for obtaining a high beam quality of $532 \mathrm{~nm}$, the beam distortion was compensated by adjusting the distribution of pumping beam in our experiment of fundamental amplification. Furthermore, better than $0.6 \mathrm{~W}$ average power, $770 \mathrm{ps}$, beam quality of $\mathrm{M}^{2}<1.2$, and $16 \mathrm{kHz}$ pulse output at $532 \mathrm{~nm}$ was obtained by a Type $\mathrm{I} \mathrm{LiB}_{3} \mathrm{O}_{5}(\mathrm{LBO})$ crystal in the critical phase matching (CPM) regime for second harmonic generation (SHG).
\end{abstract}

Keywords: laser remote sensing; photon-counting lidar; microchip laser; passively Q-switching; compact solid-state lasers

\section{Introduction}

A compact sub-nanosecond microchip laser and an amplifier currently have applications in diverse areas including ranging and imaging, micromachining, material characterization, and environmental monitoring [1-3].In particular, as transmitters, high repetition sub-nanosecond lasers have been used in photon-counting laser altimeters [4,5], or lidars, for high-resolution 2D profiles and/or 3D images of the underlying topography including soil, low-lying vegetation, water surfaces, man-made objects, ocean waves, shallow water bathymetry, 3D underwater imaging, etc. [6-8]. In a Second Generation 3D Imaging photon-counting Lidar that can be installed in an Unmanned Aerial Vehicle (UAV) developed by Sigma Space Corporation, a compact laser transmitter can deliver a $142 \mathrm{mw}$, $700 \mathrm{ps}, 532 \mathrm{~nm}$ beam at the maximum repetition rate of $22 \mathrm{kHz}$ [9]. High beam quality should be necessary because a Diffractive Optical Element (DOE) splits the $532 \mathrm{~nm}$ beam into a $10 \times 10$ array of beamlets with $80 \%$ DOE efficient was used.

In the photon-counting lidar, the laser should have high repetition, narrow pulse duration and high beam quality to satisfy the need of measuring efficiency and precision. A microchip laser and compact amplifier is the ideal selection. Lincoln Laboratory reported passively Q-switched microchip lasers using $\mathrm{Nd}^{3+}$ : YAG as the gain medium and fiber- 
pumped amplifiers that occupy a volume of less than 0.25 liters. The compact laser has pulse widths between $150 \mathrm{ps}$ and $3 \mathrm{~ns}$, pulse energies up to $250 \mu \mathrm{J}$ at $1064 \mathrm{~nm}$, and typical repetition rates between 4 and $20 \mathrm{kHz}$ [2,3]. Isyanova, Y. et al. reported a compact satelliteranging transmitter of SLR2000 consisting of a $\mathrm{Cr}^{4+}$ : YAG passively Q-switched Nd: YAG microchip laser, an air-cooled Nd: YVO4 power amplifier and a SHG module, generating $532 \mathrm{~nm}, 470 \mathrm{~mW}, 270$ ps pulses at a $2 \mathrm{kHz}$ rate [10]. Manni, J. G reported a master oscillator power amplifier (MOPA) system that combines a $1064 \mathrm{~nm}$ microchip laser oscillator with a new diode-side-pumped Nd: YVO4 zig-zag slab amplifier. A pulse energy of more than $800 \mu \mathrm{J}$ was achieved at a $2 \mathrm{kHz}$ pulse rate with the $40 \mathrm{~W}$ pump power of the amplifier [11]. Other schemes for high-repetition-rate microchip lasers have also been reported. Nicolaie, P. et al. reported an End-Pumped Composite Nd: YAG Laser Passively Q-switched by $\mathrm{Cr}^{4+}$ : YAG, and the maximum average power was $4.2 \mathrm{~W}$ with the laser operating at $24 \mathrm{kHz}$ repetition rate and a $47.8 \mathrm{~ns}$ pulse duration ( $3.7 \mathrm{~kW}$ peak power) at a beam quality of $\mathrm{M}^{2}$ $=1.34$ [12]. Pavel, N. et al. researched intracavity frequency doubling by employing a Vtype laser resonator, which yielded $532 \mathrm{~nm}$ green pulses of $226 \mu \mathrm{J}$ energy and $86 \mathrm{~ns}$ width at $4.2 \mathrm{kHz}$ [13] .

Related to the increasing of the peak power of a microchip laser, a lot of innovative progress has been reported by the institute for molecular science of Japan. Bhandari. R. et al. presented a microchip laser with $1.9 \mathrm{MW}$ peak power, $500 \mathrm{~Hz}, 550 \mathrm{ps}$, and $532 \mathrm{~nm}$ [14], which was pumped into the Quasi-Continuous Wave (QCW) regime by a fiber-coupled $120 \mathrm{~W}, 808 \mathrm{~nm}$ laser diode. Zheng, L. et al. reported a novel microchip laser using high thermal conductivity gain media with the beam of $21.5 \mathrm{~mJ}$ pulse energy, $10 \mathrm{~Hz}, 1064 \mathrm{~nm}$, and an $\mathrm{M}^{2}$ value between 10.7 and 11.8 [15]. Their research provided novel methods for the thermal restriction of microchip lasers, which would be expected in a high repetition rate.

As mentioned above, the increase in average power and corresponding pulse energies of sub-nanosecond laser with high repetition rates and high beam quality, which are an advantage of the detection distance and detection probability of lidar, is challenging in compact sizes.

In this paper, a compact sub-nanosecond master oscillator power amplifier (MOPA) laser that combines a seed based on a microchip laser, an off-axis double-pass amplifier and a SHG module is proposed. The key advantages are as follows: the seed is a freespace-pumped microchip monolithic laser without resonator optical alignment and a fiber-pumped structure; the average power and corresponding pulse energies were increased using a simple amplifier; the beam distortion of the microchip was improved without a complicated optical-shaped system.

In our work, we first designed the experiments of a monolithic microchip laser, twopass $\mathrm{Nd}$ : $\mathrm{YVO}_{4}$ amplifier and SHG. In addition, the beam distortion was compensated by adjusting the distribution of pumping light of the fundamental amplifier to be elliptic. Then, according to the fundamental results, a better than $0.6 \mathrm{~W}, 770 \mathrm{ps}, 37.5 \mu \mathrm{J}, 16 \mathrm{kHz}$, $\mathrm{M}^{2}<1.2$ beam in $532 \mathrm{~nm}$ was obtained by Type I LBO crystal in the CPM regime for SHG. Finally, we discussed the simulating results of the generation of a sub-nanosecond with the saturable absorber transmittance, the reflectivity of an output coupler and the cavity length parameter, which can be good aid for the design of a sub-nanosecond microchip laser with high repetition. On the basis of the research, we developed an engineering laser, and the dimensions of the laser head with a pump source are less than $125 \mathrm{~mm} \times 50 \mathrm{~mm}$ $\times 40 \mathrm{~mm}$.

\section{Experimental Setup}

A schematic diagram of the laser setup is shown in Figure 1. The sub-nanosecond laser consists of the microchip laser, the amplifier and the SHG. The seed was a diode endpumped passively Q-switched monolithic microchip laser with $\mathrm{YAG} / \mathrm{Nd}$ : $\mathrm{YAG} / \mathrm{Cr}^{4+}$ : YAG/YAG composite medium $(2 \mathrm{~mm} \times 2 \mathrm{~mm} \times 7 \mathrm{~mm})$. The undoped YAG, Nd: YAG $(1.1$ at. $\left.\% \mathrm{Nd}^{3+}\right), \mathrm{Cr}^{4+}: \mathrm{YAG}$, undoped YAG was bonded. The $\mathrm{Cr}^{4+}:$ YAG was used as a saturable 
absorber with $45 \%$ transmissions. Surface F1 is high-reflection coated at $1064 \mathrm{~nm}(>99.8 \%)$ and antireflection coated at $808 \mathrm{~nm}(<0.2 \%)$, while Surface F2 is an output coupler with $40 \%$ reflections of $1064 \mathrm{~nm}$. The pump source was a single emitter semiconductor laser (LD1) of $808 \mathrm{~nm}$ with a maximum $\mathrm{CW}$ of $10 \mathrm{~W}$ at $10 \mathrm{~A}$ current, and the electro-optical efficiency is about $50 \%$. The emitting wavelength of the pump source was $808 \mathrm{~nm}$ at $25^{\circ} \mathrm{C}$. The fast-axis divergence and low-axis divergence are all $8^{\circ}$ with fast-axis collimation (FAC). The heat sink of the pump source is attached to the TEC for the stabilization of the pump wavelength. A gradient-index (GRIN) lens $5 \mathrm{~mm}$ long was fixed between the LD1 and the composite crystal. L1 is a plane-convex mirror with antireflection coated at 1064 $\mathrm{nm}(<0.2 \%)$. M1 and M2, which tilted slightly, were coated with $45^{\circ}$ high-reflection at 1064 $\mathrm{nm}(>99.8 \%)$. The Beam of Seed was injected with a small incident angle to the power amplifier that consists of $\mathrm{Nd}$ : YVO4 $\left(0.5\right.$ at. $\left.\% \mathrm{Nd}^{3+}\right)$, pump shaping optics and a LD2 of $\mathrm{CW} 10 \mathrm{~W}$. The surface F3 of Nd: YVO4 was coated with antireflection layers at $1064 \mathrm{~nm}$ $(<0.2 \%)$. The opposite surface F4 of the crystal had a high reflection coating for $1064 \mathrm{~nm}$ and antireflection coating for $808 \mathrm{~nm}$. L3 and L4 are plane-convex mirrors with antireflection coating at $808 \mathrm{~nm}(<0.2 \%)$, and they were used to collimate the pump light. L2 was a cylindrical lens for adjusting the horizontal direction distribution of the pumping beam. We used a $10 \mathrm{~mm}$-long Type I LBO $\left(\theta=90^{\circ}, \beta=11.4^{\circ}\right)$ crystal (dual-band anti-reflection coating at $532 \mathrm{~nm}$ and $1064 \mathrm{~nm}$ ) for SHG due to its high enough damage threshold and relatively large angular acceptance bandwidth. The temperature of LBO was controlled by TEC for better efficiency of SHG. The $1064 \mathrm{~nm}$ beam was input to the LBO through L5, which is a plane-convex mirror with antireflection coating at $1064 \mathrm{~nm}(<0.2 \%)$ for focusing the beam of $1064 \mathrm{~nm}$. M3 was coated with $45^{\circ}$ high-reflection at $1064 \mathrm{~nm}(>99.8 \%)$ for folding the beam path. M4 and M5 are beam splitters with antireflection coating at 1064 $\mathrm{nm}(<0.2 \%)$ and partial-reflection coating at $532 \mathrm{~nm}$ for beam measurements.

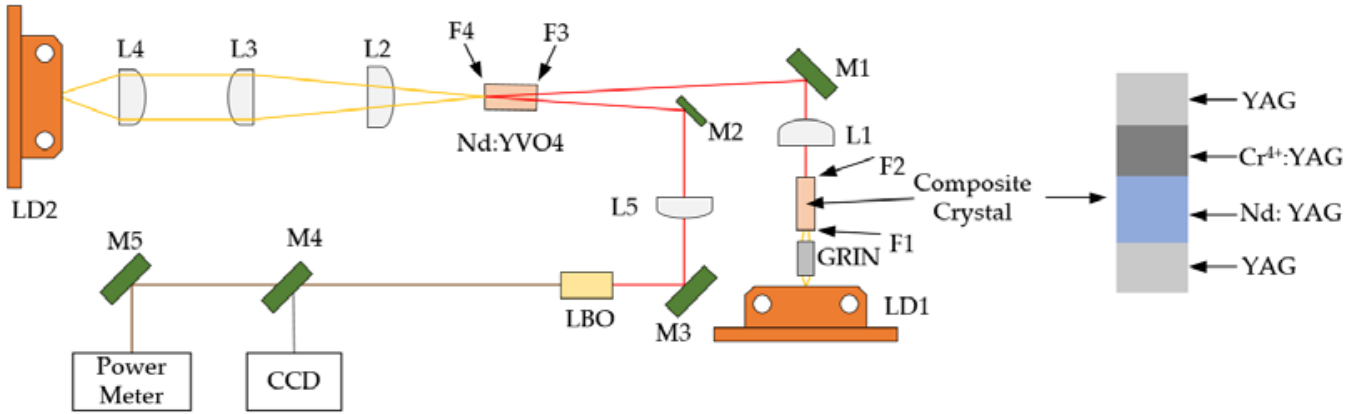

Figure 1. Schematic of the sub-nanosecond laser

\section{Experimental Results}

\subsection{Seed Source Characteristics}

In order to achieve high repetition, LD1 was run on the CW model. Considering the compact structure, a GRIN lens was selected for coupling the pump light. Both the repetition rate and outpower increased with increasing pump power as Figure 2 shows. During operation, the temperature of LD1 was controlled at $25{ }^{\circ} \mathrm{C}$ by TEC for the ideal absorption efficiency of Nd: YAG. The output average power of the seed increased from 27.8 $\mathrm{mW}$ at $2.6 \mathrm{kHz}$ to $477.2 \mathrm{~mW}$ at $21 \mathrm{kHz}$ by adding the pump power from $1.4 \mathrm{~W}$ to $4.5 \mathrm{~W}$. The average power and repetition rates were $317.8 \mathrm{~mW}$ and $16 \mathrm{kHz}$ when the pump power was at $3.9 \mathrm{~W}$. The single-pulse profile recorded by a Tektronix DPO70804C $8 \mathrm{GHz}$ Digital Phosphor Oscilloscope is shown in Fig. 3, and the full width at half-maximum (FWHM) of the laser pulse is $857 \mathrm{ps}$. 


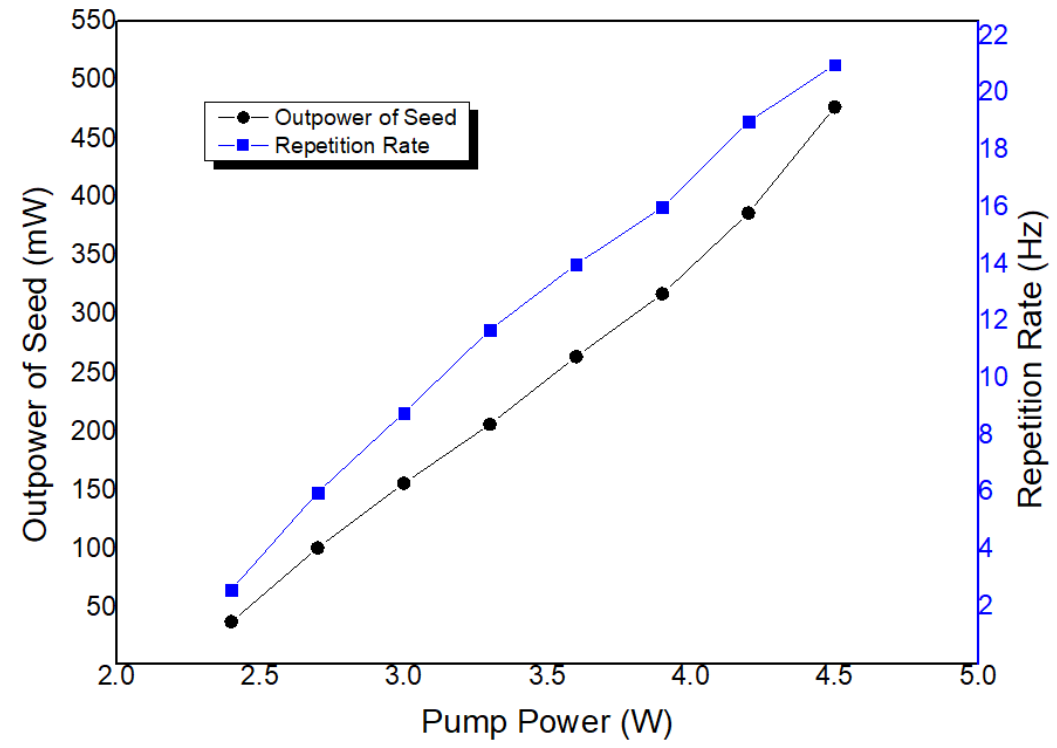

Figure 2. The average power and repetition rate of seed versus pump power.

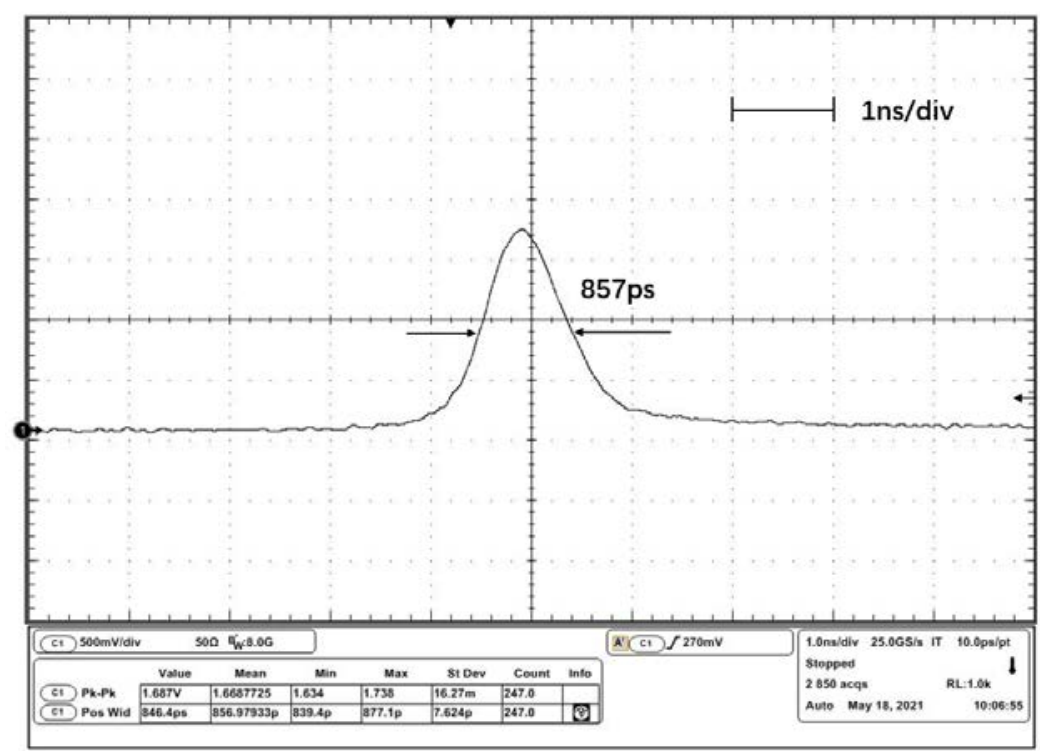

Figure 3. The single-pulse profile and pulse width of seed.

\subsection{Two-pass Amplification Characteristics}

Figure 4 shows that the outpower of the amplifier was the function of the output power of the seed and the pump power of the amplifier. The outpower of the amplifier was $1.26 \mathrm{~W}$, as the seed-injected power was $317.8 \mathrm{~mW}$ at $16 \mathrm{kHz}$ and the pump power of the amplifier was at $7 \mathrm{~W}$. The maximum output average power reached $2.3 \mathrm{~W}$, as the seedinjected power was $0.477 \mathrm{~W}$ at $21 \mathrm{kHz}$ and the pump power of the amplifier was at $9 \mathrm{~W}$. 


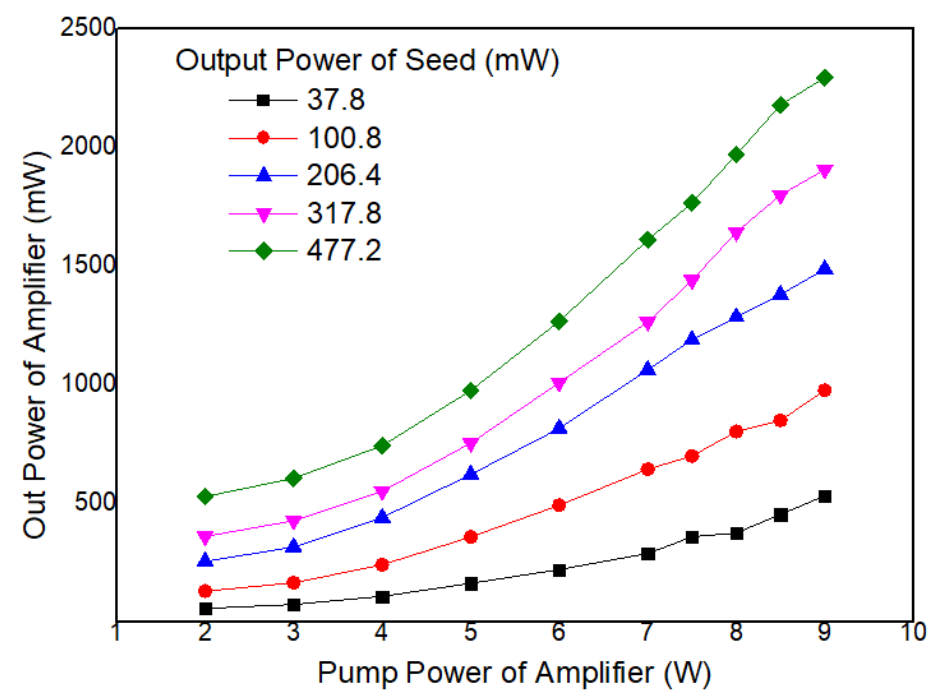

Figure 4. The amplified power of fundamental versus power of seed and input pump power of amplifier

The spatial profile of the fundamental beam was recorded with a LASERCAM HR II camera from Coherent. Figure 5 shows the beam profiles of the seed at different pump powers, and the circularity levels we measured were $0.876,0.829$, and 0.779 , corresponding to seed pump powers, respectively, of 2.4, 3.3, and $3.9 \mathrm{~W}$.

The beam profiles after amplification under different pump powers of seed as shown in Figure 6. As the pump power of amplifier was at $7 \mathrm{~W}$, the circularity levels we measured were $0.661,0.747$, and 0.927 , corresponding the pump powers of seed of $2.4 \mathrm{~W}, 3.3 \mathrm{~W}$, and $3.9 \mathrm{~W}$, respectively. After amplification, the beam distortion of the seed was compensated well. The beam profile was more circularly symmetric. The circularity of the amplified beam with a pumped power of $7 \mathrm{~W}$ versus different pump powers of seed are shown in Table 1.

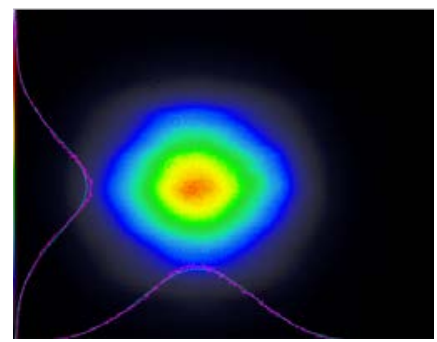

(a)

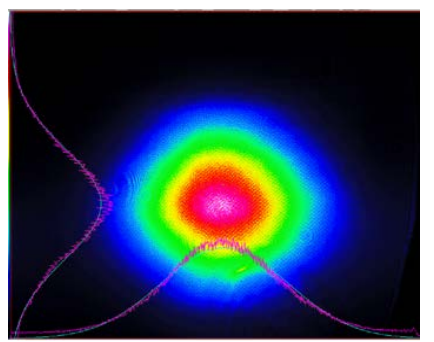

(b)

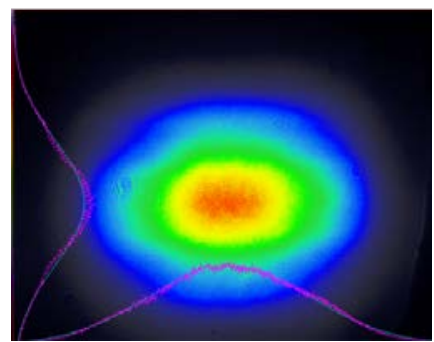

(c)

Figure 5. The beam profiles of seeds at different pump powers. (a) Beam profile of seed with pump power of $2.4 \mathrm{~W}$ (the circularity was 0.876 ). (b) Beam profile of seed with pump power of $3.3 \mathrm{~W}$ (the circularity was 0.829 ). (c) Beam profile of seed with pump power of $3.9 \mathrm{~W}$ (the circularity was 0.776 ).

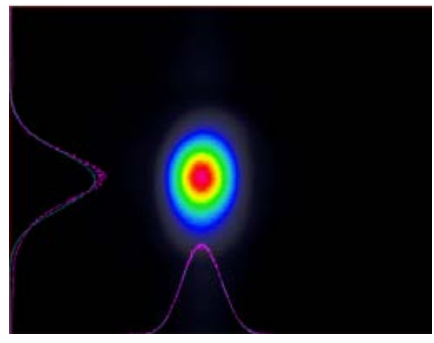

(a)

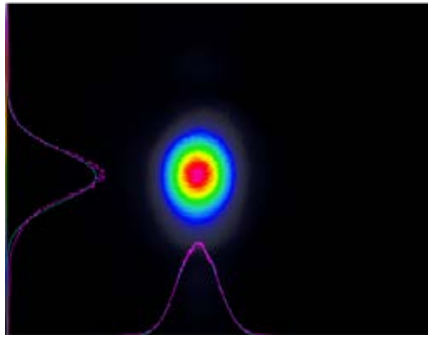

(b)

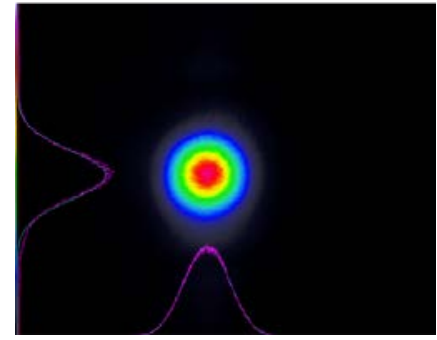

(c)

Figure 6. The amplified beam profile of the fundamental with pump power of $7 \mathrm{~W}$ at different pump powers of seed. (a) Beam profile with circularity of 0.661 , as the pump power of seed was $2.4 \mathrm{~W}$; (b) 
Beam profile with circularity of 0.747 , as the pump power of seed was $3.3 \mathrm{~W}$; (c) Beam profile with circularity of 0.927 , as the pump power of seed was $3.9 \mathrm{~W}$.

Table 1. The circularity of amplified beam with pumped power of $7 \mathrm{~W}$ versus different pump powers of seed.

\begin{tabular}{cccc}
\hline $\begin{array}{c}\text { The pump power of } \\
\text { seed }(\mathbf{W})\end{array}$ & $\begin{array}{c}\text { The output power of } \\
\text { seed }(\mathbf{m W})\end{array}$ & $\begin{array}{c}\text { Circularity of } \\
\text { seed beam }\end{array}$ & $\begin{array}{c}\text { Circularity of am- } \\
\text { plified beam }\end{array}$ \\
\hline 2.4 & 37.8 & 0.876 & 0.661 \\
3.3 & 206.4 & 0829 & 0.747 \\
3.9 & 317.8 & 0.779 & 0.927 \\
\hline
\end{tabular}

\subsection{SHG Characteristics}

Considering the limitation of volume and thermal control, we set the operating point of the amplifier at a pump power of $7 \mathrm{~W}$, as the seed-injected power was $317.8 \mathrm{~mW}$. The output average power of the fundamental was $1.26 \mathrm{~W}$. Furthermore, we focused the fundamental to a $10 \mathrm{~mm}$-long Type I LBO $\left(\theta=90^{\circ}, \beta=11.4^{\circ}\right)$ crystal for SHG. The beam of $532 \mathrm{~nm}$ had an average power of $0.6 \mathrm{~W}$ at $16 \mathrm{kHz}$. The SHG efficiency of this laser was about $47.6 \%$ from $1064 \mathrm{~nm}$ to $532 \mathrm{~nm}$. The pulse energy was $37.5 \mu \mathrm{J}$, and the peak power was about $48.7 \mathrm{~kW}$. The pulse width and repetition rate were recorded using a Tektronix DPO70804C 8 GHz Digital Phosphor Oscilloscope, and they were 769.6 ps and $16 \mathrm{kHz}$, respectively, as shown in Figure 7.

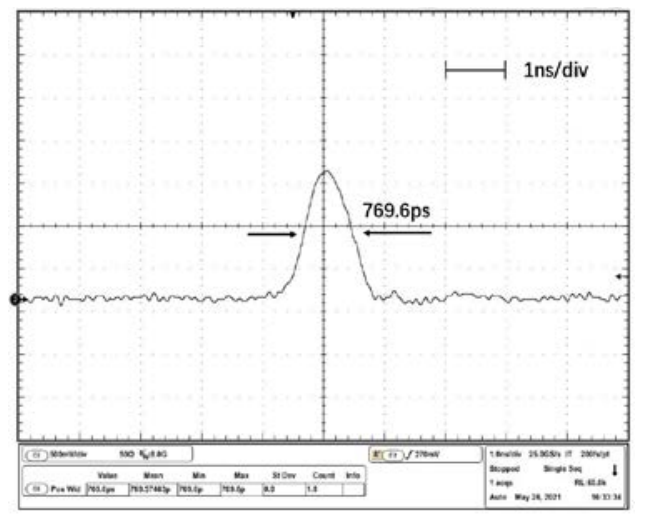

(a)

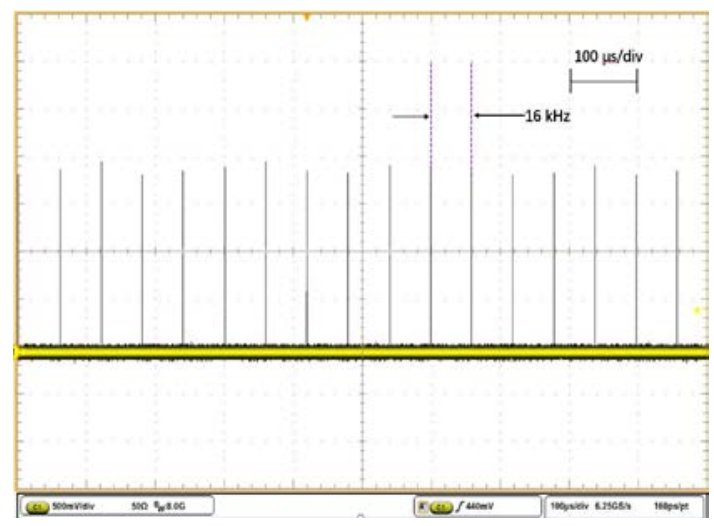

(b)

Figure 7. The pulse width and repetition rate of $532 \mathrm{~nm}$. (a) Single-pulse profile and pulse width. (b) Pulse train profile and the pulse repetition rate. 


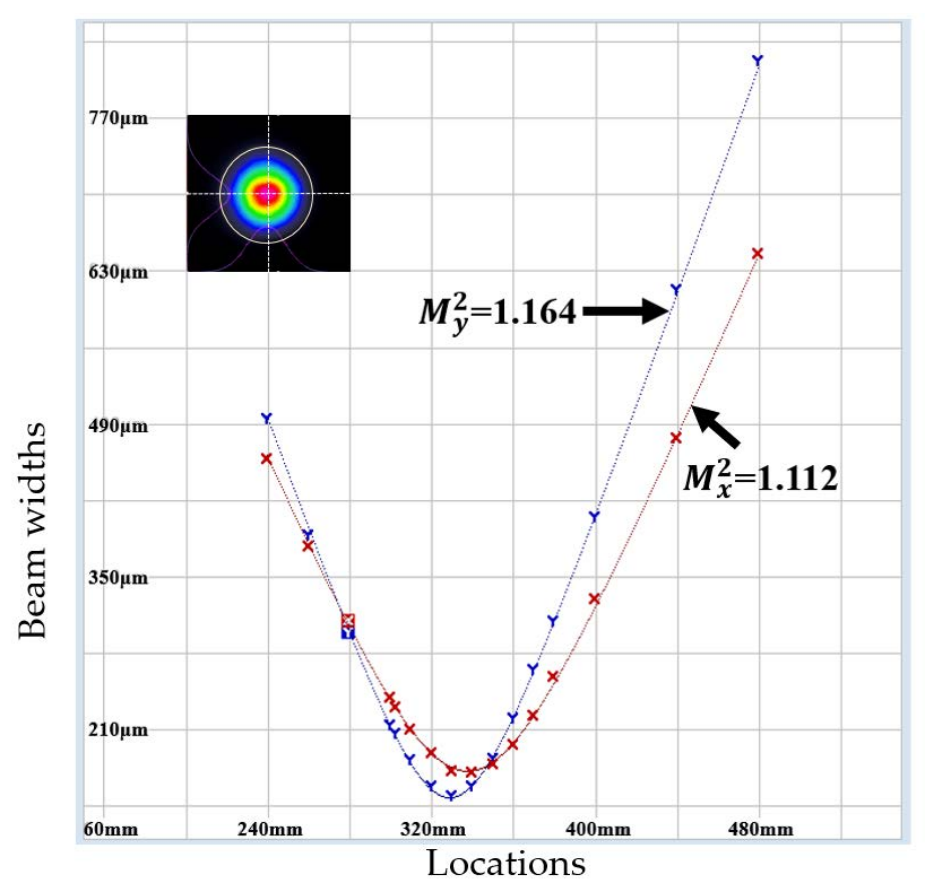

Figure 8. Quality factor $M^{2}$ of $532 \mathrm{~nm}$ beam

Quality factor $M^{2}$ of the $532 \mathrm{~nm}$ at $16 \mathrm{kHz}$ was recorded by $\mathrm{M}^{2}-200 \mathrm{~S}-\mathrm{FW}$ from OphirSpiricon with a $300 \mathrm{~mm}$-focal- length lens. The value of $M_{x}^{2}$ and $M_{y}^{2}$ were 1.112 and 1.164, respectively, as shown in Figure 8.

\section{Discussion}

\subsection{Numerical Simulation of Sub-Nanosecond}

Passively Q-switched rate equations first derived [16] by Szabo, A. and Stein, R.A., and Degnan, J.J. developed a method for optimizing the model of passively Q-switched rate equations [17]. Xiao, G. and Bass, M. presented a generalized model [18] of a passively Q-switched laser that can describe both ground and excited state absorption (ESA) at the laser wavelength of the saturable absorber. The rate equations are as follows:

$$
\begin{aligned}
\frac{d \phi}{d t}=\frac{\phi}{t_{\mathrm{r}}}\left\{2 \sigma n l-2 \sigma_{\mathrm{gs}} n_{\mathrm{gs}} l_{\mathrm{s}}-2 \sigma_{\mathrm{es}} n_{\mathrm{es}} l_{\mathrm{s}}-\left[\ln \left(\frac{1}{R}\right)+L\right]\right\} \\
\frac{d n}{d t}=-\gamma \sigma c \phi n \\
\frac{d n_{\mathrm{gs}}}{d t}=-\sigma_{\mathrm{gs}} c \phi n_{\mathrm{gs}} \\
\frac{d n_{\mathrm{es}}}{d t}=-\sigma_{\mathrm{es}} c \phi n_{\mathrm{es}} \\
n_{\mathrm{gs}}+n_{\mathrm{es}}=n_{0} \\
T_{0}=e^{-n_{0} \sigma_{\mathrm{gs}} l_{\mathrm{s}}}
\end{aligned}
$$

where $\phi$ is the density of the photon, $t_{\mathrm{r}}$ is the round trip time, and $\sigma$ is the radiation cross section of $\mathrm{Nd}^{3+}$ : YAG. $\sigma_{\mathrm{gs}}$ and $\sigma_{\mathrm{es}}$ are the ground and excited state absorption cross section, respectively. $n$ is the inverted population density in $\mathrm{Nd}^{3+}$ : YAG, while $n_{0}, n_{\mathrm{gs}}$ and $n_{\mathrm{es}}$ are the total doping particle density, and ground and excited state particle density in $\mathrm{Cr}^{4+}$ : YAG, respectively. $l$ and $l_{\mathrm{s}}$ are the lengths of $\mathrm{Nd}^{3+}$ : YAG and $\mathrm{Cr}^{4+}$ : YAG, respectively. $T_{0}$ and $R$ refer to initial transmittance of $\mathrm{Cr}^{4+}$ : YAG and reflectivity of the output coupler, respectively. $\gamma$ refers to the level degeneracy factor, while $L$ is the round trip loss in the cavity. 
Based on the above equations, we processed numerical simulation on output pulse widths with respect to key parameters as initial transmittance of $\mathrm{Cr}^{4+}$ : YAG $\left(T_{0}\right)$, reflectivity of output coupler $(R)$ and cavity length $(C L)$. The results are given by Figure 9 .

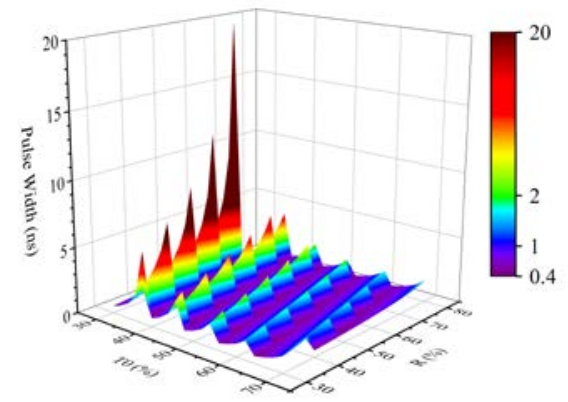

(a)

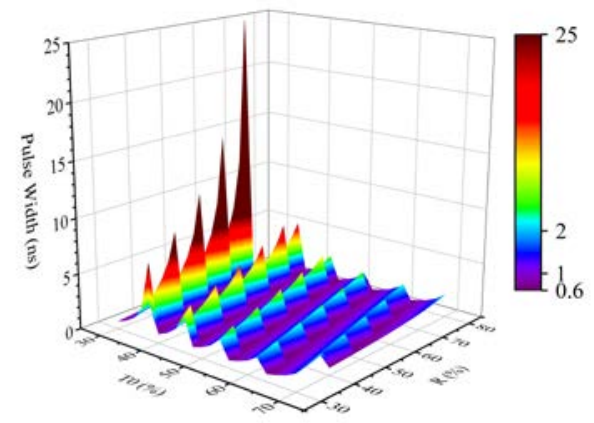

(b)

Figure 9. Pulse width change versus initial transmittance and reflectivity at different cavity lengths. (a) $C \mathrm{~L}=7 \mathrm{~mm}$; (b) $C \mathrm{~L}=9 \mathrm{~mm}$.

For obtaining the pulse width of the sub-nanosecond, there are wider range options for $T_{0}$ and $R$ as $C \mathrm{~L}$ of $7 \mathrm{~mm}$ than $C \mathrm{~L}$ of $9 \mathrm{~mm}$, as shown in Figure 9. Some key parameters are shown in Table 2. As $T_{0}=45 \%, R=40 \%$ and $C \mathrm{~L}=7 \mathrm{~mm}$, the pulse width of the seed was expected to be about 850 ps. This resulted in a good agreement between the experiments and modeling.

Table 2. Pulses width results for cavity lengths with $45 \%$ initial transmittance of $\mathrm{Cr}^{4+}$ : YAG

\begin{tabular}{ccc}
\hline Cavity length, $\boldsymbol{C L} \mathbf{( m m )}$ & $\begin{array}{c}\text { Reflectivity of } \\
\text { output coupler, } \boldsymbol{R}^{\mathbf{m}}\end{array}$ & $\begin{array}{c}\text { Pulse width } \\
\text { (ns) }\end{array}$ \\
\hline \multirow{2}{*}{7} & 35 & 0.909 \\
& 40 & 0.851 \\
9 & 45 & 0.809 \\
& 35 & 1.169 \\
& 40 & 1.095 \\
& 45 & 1.041 \\
\hline
\end{tabular}

\subsection{Compact Design and Beam Distortion Compensation}

\subsubsection{Compact Design}

For realizing a compact engineering laser, we used two single-emitter semiconductor lasers as pump sources that remove the limitations of the fiber [19]. In addition, we used a GRIN lens and composite crystal in the seed source, which are tiny and robust. Then, the isolator was not necessary because the off axis two-pass amplification was adopted. Finally, the compact laser head occupying a volume of less than 0.25 liters was realized.

\subsubsection{The Compensation of Beam Distortion}

With the increase in pump power, the beam distortion of the seed was severe because of the increase in thermal loading. The distribution of the seed beam was divergent in the horizontal direction for obtaining the ideal distribution of the fundamental. The cylindrical lens L2 was used for adjusting the pump distribution of the amplifier in the horizontal direction. We can conclude the pump distribution of the amplifier was about orthogonal to the distribution of the seed beam, since the pump power of the amplifier was constant. 
The beam distortion of the seed was compensated well owing to the restraint of the horizontal direction amplification and the enhanced vertical direction, as the pump power of the seed was 3.9 W. Therefore, in this experiment of amplification, the regulation of the pump distribution of the amplifier was effective in compensating the thermal-induced distortion of seed beam. It would be helpful for the design of a high-beam-quality laser with a compact size.

\section{Conclusions}

In this study, we demonstrated a compact sub-nanosecond $532 \mathrm{~nm}$ laser with an average power of $0.6 \mathrm{~W}$ corresponding to the LD pumped whole power of $10.9 \mathrm{~W}$, repetition rate of $16 \mathrm{kHz}$, pulse duration of $769 \mathrm{ps}$, and pulse energy of $37.5 \mu \mathrm{J}$, and the beam was characterized by $M_{x}^{2}=1.112$, and $M_{y}^{2}=1.164$. A MOPA system based on a passively Qswitched monolithic microchip laser was displayed in the experiment. However, the ideal average power of the microchip laser in the experiment less than $317.8 \mathrm{~mW}$, which was restricted by the thermal loading. We proposed the power amplification and beam distortion compensation approaches in our experiments. The lidar with a high average power and corresponding pulse energies of the transmitter is an advantage of the detection distance and detection probability. The high beam quality contributes to increasing the efficiency of the transmitting optical system. Considering its high performance and compact characteristics, the reported sub-nanosecond laser can be widely used.

Author Contributions: Conceptualization, D.-D.M., M.Z. and Z.-W.F.; methodology, D.-D.M.; simulation, T.-Q.W. experiment, M.Z. and T.-Q.W.; validation, Z.-D.Q. and X.-L.L.; supervision, Z.W.F.; writing - original draft preparation, D.-D.M.; writing-review and editing, D.-D.M. and X.L.L. project administration, D.-D.M. All authors have read and agreed to the published version of the manuscript.

Funding: This research was funded by the National Key Research and Development Program of China (2020YFE0204600) and Key Research and Development Program of Tianjin (19YFZCGX00210).

Conflicts of Interest: “The authors declare no conflicts of interest."

\section{References}

1. Zayhowski, J. Topical Papers on Microchip Lasers and Applications. Passively Q-Switched Microchip Lasers and Applications. The Review of Laser Engineering 1998, 26, 841-846.

2. Zayhowski, J.J.; Terry, J.; Clarkson, W.A. Compact solid state sources and their applications. Proc Spie 2004, 5620, 155-169.

3. Zayhowski, J.J.; Dill, C.; Cook, C.; Daneu, J.L. Mid- and High-Power Passively Q-Switched Microchip Lasers. In Proceedings of the Advanced Solid State Lasers, Boston, Massachusetts, 1999/01/31, 1999; p. TuC1.

4. Degnan, J. Photon-counting multikilohertz microlaser altimeters for airborne and spaceborne topographic measurements. Journal of Geodynamics 2002, 34, 503-549, doi:10.1016/S0264-3707(02)00045-5.

5. Degnan, J.J. Unified Approach to Photon-Counting Microlaser Rangers, Transponders, and Altimeters. Surveys in Geophysics 2001, 22, 431-447, doi:10.1023/A:1015659931843.

6. Albota, M.A.; Heinrichs, R.M.; Kocher, D.G.; Fouche, D.G.; Player, B.E.; O'Brien, M.E.; Aull, B.F.; Zayhowski, J.J.; Mooney, J.; Willard, B.C.; et al. Three-dimensional imaging laser radar with a photon-counting avalanche photodiode array and microchip laser. Applied optics 2002, 41, 7671-7678, doi:10.1364/ao.41.007671.

7. Degnan, J.J. Rapid, Globally Contiguous, High Resolution 3D Topographic Mapping of Planetary Moons Using a Scanning, Photon-Counting Lidar. In Proceedings of the International Workshop on Instrumentation for Planetary Missions, October 01, 2012, 2012; p. 1086. 
8. Degnan, J.; Machan, R.; Leventhal, E.; Jodor, G.; Field, C. Photon-Counting, 3D imaging Lidars Operating at Megapixels per Second. In Proceedings of the Conference on Lasers and Electro-Optics/International Quantum Electronics Conference, Baltimore, Maryland, 2009/05/31, 2009; p. CFJ6.

9. Degnan, J.; Wells, D.; Machan, R.; Leventhal, E. Second generation airborne 3D imaging lidars based on photon counting - art. no. $67710 N ; 2007$.

10. Isyanova, Y.; Wall, K.; Flint, J.; Moulton, P.; Degnan, J. High-Power, Short-Pulse, Compact SLR2000 Laser Transmitter. 2004, doi:10.1364/ASSP.2004.MB14.

11. Manni, J. Amplification of microchip oscillator emission using a diode-pumped wedged-slab amplifier. Optics Communications - OPT COMMUN 2005, 252, 117-126, doi:10.1016/j.optcom.2005.03.038.

12. Pavel, N.; Saikawa, J.; Kurimura, S.; Taira, T. High Average Power Diode End-Pumped Composite Nd:YAG Laser Passively Q-switched by Cr4+:YAG Saturable Absorber. Japanese Journal of Applied Physics 2001, 40, 1253-1259, doi:10.1143/JJAP.40.1253.

13. Pavel, N.A.; Saikawa, J.; Dascalu, T.; Lupei, V.; Taira, T. All-solid-state diode and end-pumped Nd:YAG laser passively Qswitched by Cr 4+ :YAG saturable absorber. Proceedings of SPIE 2004, 5581, 170-179.

14. Bhandari, R.; Taira, T. High Repetition Rate MW Peak Power at 532 nm Using Microchip Laser. In Proceedings of the CLEO: 2013, San Jose, California, 2013/06/09, 2013; p. JW2A.26.

15. Zheng, L.; Kausas, A.; Taira, T. >30 MW peak power from distributed face cooling tiny integrated laser. Optics express 2019, 27, 30217-30224, doi:10.1364/oe.27.030217.

16. Szabo, A.; Stein, R. Theory of Laser Giant Pulsing by a Saturable Absorber. Journal of Applied Physics 1965, 36, 1562-1566, doi:10.1063/1.1703087.

17. Degnan, J.J. Optimization of passively Q-switched lasers. IEEE Journal of Quantum Electronics 1995, 31, 1890-1901, doi:10.1109/3.469267.

18. Xiao, G.; Bass, M. A generalized model for passively Q-switched lasers including excited state absorption in the saturable absorber. IEEE Journal of Quantum Electronics 1997, 33, Medium: X; Size: pp. 41-44, doi:10.1109/3.554875.

19. Bhandari, R.; Ishigaki, N.; Uno, S.; Hiroki, T.; Saikawa, J.; Tojo, K.; Taira, T. Novel End-Pumping Method for Stable and Compact Microchip Laser. In Proceedings of the 2017 European Conference on Lasers and Electro-Optics and European Quantum Electronics Conference, Munich, 2017/06/25, 2017; p. CA_P_34. 